\title{
Object identification using correlated orbital angular momentum states
}

\author{
Néstor Uribe-Patarroyo, ${ }^{1}$, Andrew Fraine, ${ }^{1}$ David S. Simon, ${ }^{1,2}$ Olga Minaeva, ${ }^{3}$ and Alexander V. Sergienko ${ }^{1,4}$ \\ ${ }^{1}$ Dept. of Electrical and Computer Engineering, Boston University, 8 Saint Marys St., Boston, MA 02215, USA \\ ${ }^{2}$ Dept. of Physics and Astronomy, Stonehill College, 320 Washington St., Easton, MA 02357, USA \\ ${ }^{3}$ Dept. of Biomedical Engineering, Boston University, 44 Cummington St., Boston, MA 02215, USA \\ ${ }^{4}$ Dept. of Physics, Boston University, 590 Commonwealth Avenue, Boston, MA 02215, USA
}

\begin{abstract}
Using spontaneous parametric down conversion as a source of entangled photon pairs, correlations are measured between the orbital angular momentum (OAM) in a target beam (which contains an unknown object) and that in an empty reference beam. Unlike previous studies, the effects of the object on offdiagonal elements of the OAM correlation matrix are examined. Due to the presence of the object, terms appear in which the signal and idler OAM do not add up to that of the pump. Using these off-diagonal correlations, the potential for high-efficiency object identification by means of correlated OAM states is experimentally demonstrated for the first time. The higher-dimensional OAM Hilbert space enhances the information capacity of this approach, while the presence of the off-diagonal correlations allows for recognition of specific spatial signatures present in the object. In particular, this allows the detection of discrete rotational symmetries and the efficient evaluation of multiple azimuthal Fourier coefficients using fewer resources than in conventional pixel-by-pixel imaging. This represents a demonstration of sparse sensing using OAM states, as well as being the first correlated OAM experiment to measure properties of a real, stand-alone object, a necessary first step toward correlated OAM-based remote sensing.
\end{abstract}

\section{Introduction}

Correlated optical sensing uses various types of correlations between pairs of photons or pairs of classical light beams to form an image or to detect specific object features. This includes techniques such as ghost imaging [14] and compressive ghost imaging [6, 7]. In ghost imaging, correlated light is sent through two different paths, one of which contains the target object and a bucket detector, and the other has a spatially-resolving detector but no object. Correlation between the outputs of each detector allows reconstruction of an image or sensing of particular features of the object [8]. In compressive sensing [5], the illumination of the target object is modulated by a number of known (usually random) spatially-varying transmission masks and the output is collected by a bucket detector. An estimation of the object or its features can be found by correlating the intensity of the detected light with the mask profile. Compressive ghost imaging uses the techniques of compressive sensing in a ghost imaging setup to attain more efficient imaging, such as the recently proposed multipleinput ghost imaging [9], in which the bucket detector is replaced by a sparse-pixelated detector. The importance of compressive sensing stems from the fact that successful sensing of the object requires a number of measurements much smaller than the image size (measured in number of pixels), which has important implications for more efficient remote-sensing techniques.

The use of orbital angular momentum (OAM) states in classical and quantum imaging techniques has been shown to provide additional effects that enhance the sensitivity to particular features of an object. For example, it has been shown that the use of OAM modes in phase imaging configurations increases edge contrast by using a spiral phase distribution as a filter [8]. In addition, digital spiral imaging [10, 11] has been proposed as a technique in which the OAM basis is used to illuminate the object and to analyze the transmitted or reflected light. The twodimensional spatial structure of the mode along with the high dimension of the OAM basis set leads to the probing of two-dimensional objects without obtaining a pixel by pixel reconstruction, analogous to the approach of compressive sensing.

This high dimensionality combined with the use of correlated two-photon states gives rise to an improved version of digital spiral imaging which exploits the full twodimensional OAM joint spectrum. This new method [12], called correlated spiral imaging (CSI), is potentially suitable for OAM-based remote sensing. The two-photon joint OAM spectrum provides object information concerning spatial symmetries, yielding more efficient object recognition than conventional imaging and sensing techniques. CSI can be viewed as similar to compressive sensing, in the sense that sampling is performed in randomly-chosen elements of a high-dimensional OAM basis, so that if the object has a compact representation in this basis, a small number of measurements will suffice to identify it.

This letter presents, to the best of our knowledge, the first experimental demonstration of a sparse sensing technique based on OAM states. It uses a simplified CSI implementation that expands states generated by spontaneous parametric down conversion (SPDC) [13-15] in terms of the OAM basis in order to identify objects without attempting image reconstruction. Significant information from the object is available by measuring the joint OAM spectrum of twophoton states, giving rise to novel features outside the main OAM-conserving diagonal [16], due to the interaction with the object. In order to use this information for object identification, the relationship of the joint OAM spectrum to the azimuthal Fourier coefficients is derived. In the field of 
remote sensing this capability enables efficient object identification without the requirement of a full two-dimensional image. The present demonstration also represents the first correlated OAM-based sensing experiment representative of a practical remote-sensing setup, in which physical objects completely detached from any optical component are used as targets for identification. All previous experiments on correlated sensing using OAM states ([8] for example) have used simulated objects drawn on a spatial light modulator (SLM). High sensitivity to the spatial properties of the targets is obtained, due to the high amount of information per photon attainable by the use of OAM states.

\section{Theoretical basis}

Correlated OAM states for the implementation of CSI are created through the process of spontaneous parametric down conversion (SPDC). The two-photon state produced by SPDC can be expressed as an expansion in LaguerreGauss (LG) modes $|l, p\rangle[14]$ defined as

$$
\langle r, \phi \mid l, p\rangle=k_{p}^{|l|} r^{|l|} e^{-r^{2} / w_{0}^{2}} \mathrm{~L}_{p}^{|l|}\left(2 r^{2} / w_{0}^{2}\right) e^{i \phi l},
$$

where $k_{p}^{|l|}$ is a normalization constant, $L_{n}^{\alpha}(x)$ is the generalized Laguerre polymonial of order $n$ and $w_{0}$ is the beam waist as defined in this plane. The azimuthal phase term in the LG expression gives rise to modes with quantized OAM of order $l$. Discrimination between individual radial modes $p \neq 0$ is not attainable by current experimental techniques [17], therefore only the case $p=0$ will be considered for simplicity and the $p$ index will be dropped hereafter. The following derivation is easily generalized for nonzero $p$. In the case of detecting LG modes by the use of holograms and mono-mode fibers [16], the cross-talk given by modes with $p \neq 0$ should be taken into account [14]. In the case of a detection scheme with sensitivity to all $p$ modes [17], projections should consider both the $l$ and $p$ indices.

The total OAM content is known to be conserved in a collinear SPDC process [16], and in the case of a Gaussian pump (OAM content $l_{p}=0$ ) the two-photon state can be written as

$$
|\Psi\rangle=\sum_{l=-\infty}^{\infty} C_{l}|-l\rangle|l\rangle,
$$

with $C_{l}$ given by the phase-matching condition and the relation between the pump, signal and idler beam waists $[12,14] . C_{l}$ can be viewed as the natural OAM spectrum coefficients from SPDC.

Consider the SPDC setup of Fig. 1, where a correlated OAM state is generated after the collinear down-converted beam enters a non-polarizing beam splitter (BS). After splitting, one branch corresponds to the reference arm and the other branch has the object in its path. The stand-alone object is detached from any optical component. The correlated state analyzer is composed of two identical detection

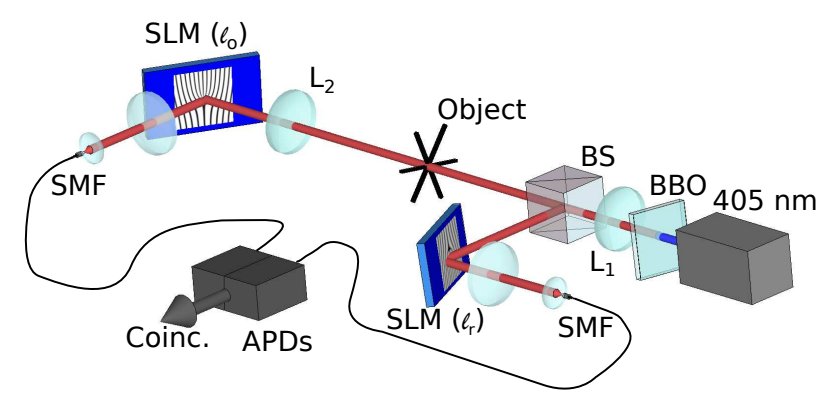

FIG. 1. (Color online) Setup for the sensing of the object via orbital angular momentum of correlated photons.

systems used to make the joint projection on the LG basis. The detectors are connected to a coincidence circuit that analyzes light with OAM number $l_{r}$ in the reference arm and $l_{o}$ in the object arm. The coincidence rate is proportional to the joint spectrum $P\left(l_{r}, l_{o}\right)$ of the down-converted beam after interaction with the object. This setup assumes the object is a transmissive target; it is sufficient to reposition the detection unit containing lens L2 in order to instead collect light in reflective mode from the target.

The object is described by a spatially varying amplitude and/or phase function $A(r, \phi)$. Interaction of an LG state with the object can be expressed as an operator in the LG basis by the matrix elements of the operator $A$

$$
\langle k|A| l\rangle=A_{k l} \text {. }
$$

One can calculate $\langle k|A| l\rangle$ (with $\rho=\sqrt{2} r / w_{0}$ ) using

$$
\begin{aligned}
& \langle k|A| l\rangle=\sqrt{\frac{2}{\pi|k| !}} \sqrt{\frac{2}{\pi|l| !}} \times \\
& \frac{1}{2} \int_{0}^{\infty} d \rho \int_{0}^{2 \pi} d \phi \rho^{|k|+|l|+1} e^{-\rho^{2}} e^{-i \phi(k-l)} A(\rho, \phi) .
\end{aligned}
$$

By performing the integration in the radial direction the function $R_{k l}(\phi)$ is obtained, which depends only on the azimuthal variable

$$
R_{k l}(\phi)=\frac{2}{\sqrt{|k| !|l| !}} \int_{0}^{\infty} d \rho \rho^{|k|+|l|+1} e^{-\rho^{2}} A(\rho, \phi) .
$$

This integration reveals that $R_{k l}(\phi)$ corresponds to the azimuthal features of $A(\rho, \phi)$ at a weighted average of object radius. The weight function is the squared absolute value of an LG mode $|l|+|k|$, which is an annular function with its maximum at a radius $w_{0} \sqrt{(|l|+|k|) / 2}[18]$.

The second integration has the form (with $m=k-l$ )

$$
\langle k|A| l\rangle=\frac{1}{2 \pi} \int_{0}^{2 \pi} d \phi e^{-i m \phi} R_{k l}(\phi) .
$$

Therefore, $A_{k l}$ is the $m$-th coefficient of the Fourier series of $R_{k l}(\phi)$, the region selected by the "ring" in the first integral. In the setup of Fig. 1, after interaction with the object 
the two-photon state transforms into (dropping the limits of the summations)

$$
\left|\Psi^{\prime}\right\rangle=\sum_{l} C_{l}|-l\rangle[A|l\rangle]=\sum_{l} C_{l}|-l\rangle\left[\sum_{k^{\prime}} A_{k^{\prime} l}\left|k^{\prime}\right\rangle\right] .
$$

It follows that the joint spectrum is given by

$$
P\left(l_{r}, l_{o}\right)=\left\langle l_{r}\right|\left\langle l_{o}|| \Psi^{\prime}\right\rangle=C_{l_{r}} A_{l_{r}, l_{o}},
$$

which contains information about the natural SPDC OAM spectrum coefficients $C_{l}$ as well as information about the object $A_{k l}$. The OAM spectral signature of the object can be isolated by performing $A_{k l}=P(l, k) / C_{l}$ for $C_{l} \neq 0$.

States with $l_{r}+l_{o} \neq l_{p}$ can have non-zero probability. When there was no object present, these states were forbidden by OAM conservation [13-17]; they represent the interaction of the correlated OAM state from SPDC with the object. These new non-conservation elements have a contribution to its total OAM content from the object $m=l_{o}+l_{r}-l_{p}$, and carry direct information of the $m$-fold rotational symmetries of the object at a $\left|l_{r}\right|+\left|l_{o}\right|$ radius. For instance, considering a Gaussian pump $\left(l_{p}=0\right)$, the measured joint spectrum with a $m$-fold rotationally symmetric target will show strong components with total OAM content $\pm m$, and higher harmonics with lower amplitudes at $n m,|n|>1$ being $n$ an integer.

\section{Experiment}

The experimental setup schematic is shown in Fig. 1 A $1.5 \mathrm{~mm}$-thick BBO crystal is pumped with a $30 \mathrm{~mW}$ diode laser at $405 \mathrm{~nm}$. To measure the OAM spectrum, an SLM is used to display computer generated holograms. The holograms change the winding number $l$ of LG light, while two lenses and a mono-mode fiber selectively couple modes with $l=0$ [14]. One condition for obtaining $A_{k l}$ without any post-processing is that the projections of Eqs. (4) and (8) are made in equivalent planes, i.e. planes that are imaged onto one another. In the current setup, which is a representative demonstration of remote sensing, lens L2 images an object at a fixed distance onto the plane of the SLM. In a general remote sensing application, L2 will be placed appropriately so that all targets will be at distances greater than the hyperfocal length of L2. Therefore, all objects will be imaged in focus onto the SLM plane.

Fig. 2 (a-b) illustrates the measurement of the natural SPDC OAM joint spectrum $C_{l}$. The sign of $l_{r}$ is flipped to compensate for the odd number of mirrors in order to reflect the OAM conservation in SPDC. In the absence of an object, only the main diagonal terms which match the total OAM content of the pump appear, without any nondiagonal terms observed [15]. Experimental data represent the mean of four measurements, from which the standard deviation is calculated and displayed as error bars. Fig. 2

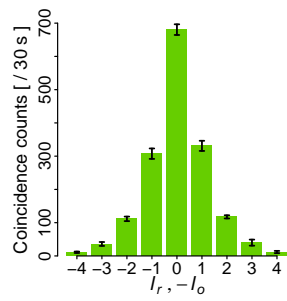

(a)

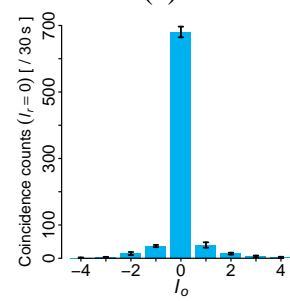

(c)

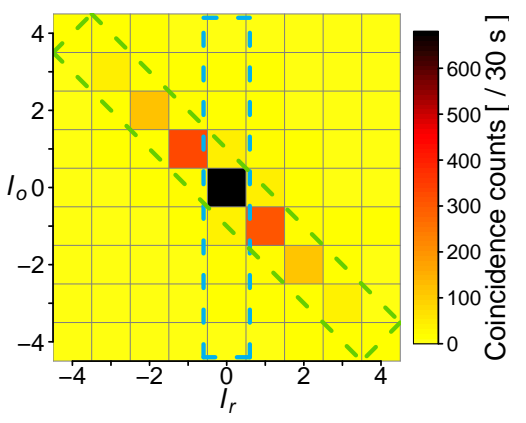

(b)
FIG. 2. (Color online) (a) Histogram of the main diagonal and (b) complete two-dimensional OAM joint spectrum for our configuration with no object. (c) Corresponding $P\left(l_{o} \mid l_{r}=0\right)$ cross section. The blue (vertical) box in the joint spectrum denotes the section in (a) and the green (diagonal) box denotes the section in (c).

(c) shows the histogram of the diagonal and the cross section for $P\left(l_{o} \mid l_{r}=0\right)$.

To demonstrate the capability and high sensitivity of this new technique for object recognition, the joint spectra using targets with different rotational symmetries are analyzed. As seen in (8), an object can impart extra features in the joint spectrum according to its azimuthal Fourier series at different radii. In particular, an object with four-fold rotational symmetry will have strong signatures corresponding to a total OAM content of \pm 4 . To fit the scale of our setup, opaque strips $175 \mu \mathrm{m}\left(0.83 w_{0}\right)$ thick, are placed in the object arm arranged with specific rotational symmetries. These targets behave as transmission masks that block light, and the integration time is adjusted in order to have a similar amount of counts with respect to the case of no object. Test objects with well-defined dominant fourand six-fold symmetries shown in Figs. 3 (a) and 4 (a) considerably modify the joint spectra [Figs. 3 (b) and 4 (b)] by adding extra diagonals with total OAM content of $l= \pm 4$ and $l= \pm 6$, respectively. The two objects are clearly distinguished by the unique features of their respective joint spectrum.

\section{Discussion}

More insight about the structure of each object is acquired by analyzing specific non-diagonal cross sections such as the two in Figs. 3 (d) and 4(d). Previous works regarding the OAM joint spectrum of SPDC [12-15, 17] have considered only the main diagonal elements corresponding to the conservation of OAM with respect to the 


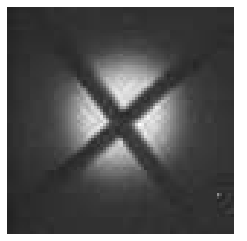

(a)

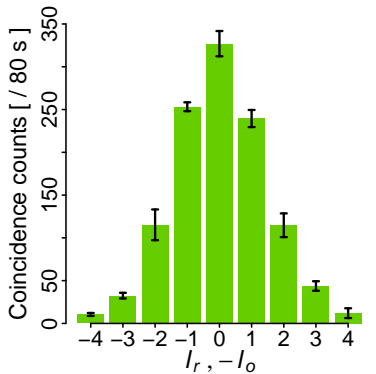

(c)

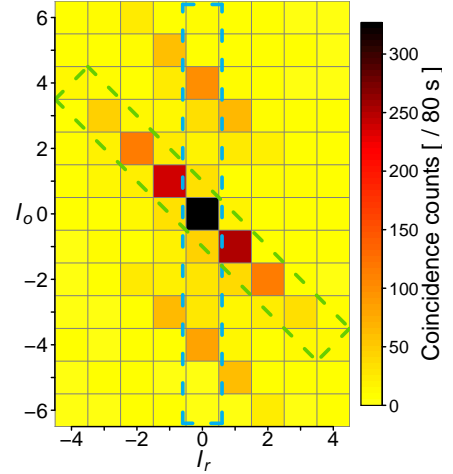

(b)

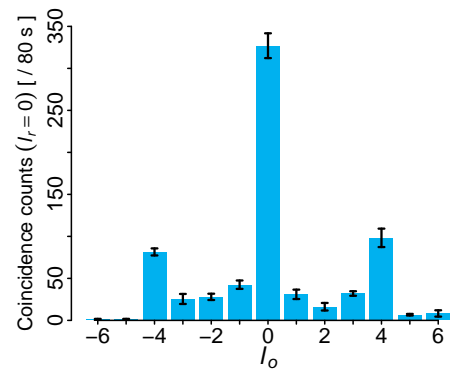

(d)
FIG. 3. (Color online) (a) Image of the cross used as target, (b) the experimental joint spectrum, (c) a histogram of the joint spectrum main diagonal and (d) $P\left(l_{o} \mid l_{r}=0\right)$ cross section of the joint spectrum.

pump [Figs. 3 (c) and 4(c)]. In the current case, there is an interaction between the OAM from SPDC and the spatial features of the object resulting in contributions which require analysis of the complete two-dimensional joint spectrum.

The non-diagonal cross section $P\left(l_{o} \mid l_{r}=0\right)$ shown in Fig. 3 (d) indicates significant four-fold symmetry as the dominant feature of the object. This feature can be uniquely attributed to the object because it is outside the diagonal that corresponds to the conservation of OAM in SPDC. Fig. 4 (d), showing the same non-diagonal cross section for the six-fold symmetric object, exhibits richer features associated with the higher complexity of the object. Specifically, apart from the dominant six-fold symmetric contribution, a three-fold contribution is observed. At the center of the object [Fig. 4(a)], the three stripes are displaced with respect to the center. This forms a triangular shape in a small region with three-fold rotational symmetry. This small deviation from a strict six-fold symmetry is readily observed in the non-diagonal cross section by the appearance of significant contributions at $l_{o}= \pm 3$.

Although the cross section $P\left(l_{o} \mid l_{r}=0\right)$ is a good indicator of the presence of different symmetries, it is necessary to analyze the full two-dimensional OAM joint spectrum to extract the relative contribution of each symmetry component. For example, Fig. 4 (d) indicates a relative contribution of six-and three-fold symmetries at a ratio of

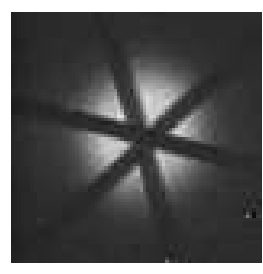

(a)

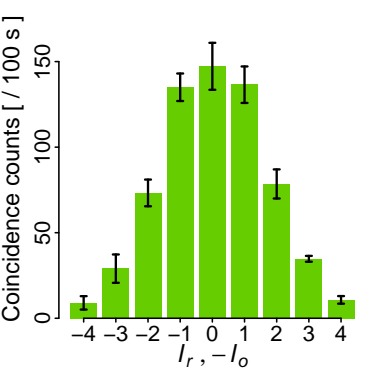

(c)

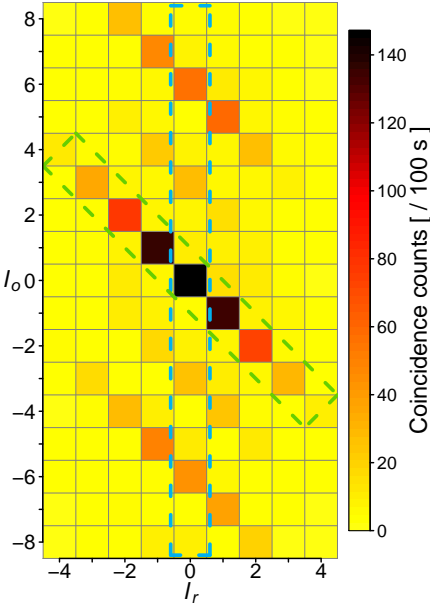

(b)

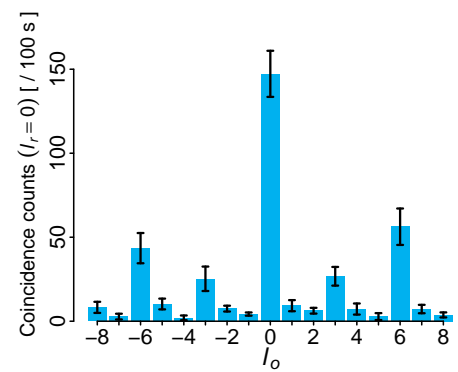

(d)
FIG. 4. (Color online) (a) Image of the three-arm cross used as target, (b) the experimental joint spectrum, (c) a histogram of the joint spectrum main diagonal and (d) $P\left(l_{o} \mid l_{r}=0\right)$ cross section of the joint spectrum.

approximately $2: 1$. However, to judge the relative size of the areas within the object that exhibit each symmetry, the total contribution of the entire diagonals should be considered. In this case, the joint spectrum in Fig. 4(b) clearly shows that the region with three-fold symmetry is significantly smaller than the region with six-fold symmetry.

As a final note, it should be pointed out that rotating the object about the azimuthal axis simply shifts the phase of the field by a constant, which will have no effect on the correlation rate. Therefore, if an object is rotated, the method will correctly continue to identify it as the same object.

In summary, a practical demonstration of high-efficiency sensing using OAM states has been presented which allows recognition of objects using a small number of measurements. These results demonstrate that an object imprints its own characteristic features onto the joint OAM joint spectrum of SPDC as predicted by Eq. (8). New elements arise that do not fulfill the OAM conservation condition required by SPDC alone, but which are affected by the object as well. The capability of CSI for object recognition is demonstrated by these results including high sensitivity to small symmetry components. This represents a realistic remote sensing application using physical objects detached 
from any optical components. Although the experiment was conducted with a transmissive object, a trivial modification of the optical setup (simply changing the position of the lens, SLM, and object arm detector) makes it suitable for remote sensing of reflective targets at arbitrarily large distances.

\section{Acknowledgements}

This research was supported by the DARPA InPho program through US Army Research Office award W911NF10-1-0404.

* uribepnr@bu.edu

[1] D. N. Klyshko, "Effect of focusing on photon correlation in parametric light scattering," Sov. Phys. JETP 67, 1131 (1988).

[2] A. V. Belinskii and D. N. Klyshko, "Two-photon optics: diffraction, holography, and transformation of twodimensional signals," Sov. Phys. JETP 78, 259 (1994).

[3] T. B. Pittman, Y. H. Shih, D. V. Strekalov, and A. V. Sergienko, "Optical imaging by means of two-photon quantum entanglement," Phys. Rev. A 52, R3429-R3432 (1995)

[4] Ryan S. Bennink, Sean J. Bentley, and Robert W. Boyd, "'Two-Photon' coincidence imaging with a classical source," Phys. Rev. Lett. 89, 113601 (2002)

[5] E.J. Candes and M.B. Wakin, "An introduction to compressive sampling," Signal Processing Magazine, IEEE 25, 21 -30 (2008)

[6] Ori Katz, Yaron Bromberg, and Yaron Silberberg, "Compressive ghost imaging," Applied Physics Letters 95, 131110 (2009)
[7] Vladimir Katkovnik and Jaakko Astola, "Compressive sensing computational ghost imaging," J. Opt. Soc. Am. A 29, 1556-1567 (2012).

[8] B. Jack, J. Leach, J. Romero, S. Franke-Arnold, M. RitschMarte, S. M. Barnett, and M. J. Padgett, "Holographic ghost imaging and the violation of a Bell inequality," Phys. Rev. Lett. 103, 083602 (2009)

[9] Wenlin Gong and Shensheng Han, "Multipleinput ghost imaging via sparsity constraints," J. Opt. Soc. Am. A 29, 1571-1579 (2012).

[10] Lluis Torner, Juan Torres, and Silvia Carrasco, "Digital spiral imaging," Opt. Express 13, 873-881 (2005)

[11] Gabriel Molina-Terriza, Liis Rebane, Juan P. Torres, Lluis Torner, and Silvia Carrasco, "Probing canonical geometrical objects by digital spiral imaging," Journal of the European Optical Society: Rapid Publications 2 (2007),

[12] David S. Simon and Alexander V. Sergienko, "Two-photon spiral imaging with correlated orbital angular momentum states," Phys. Rev. A 85, 043825 (2012)

[13] J. P. Torres, A. Alexandrescu, and Lluis Torner, "Quantum spiral bandwidth of entangled two-photon states," Physical Review A 68, 050301 (2003)

[14] Xi-Feng Ren, Guo-Ping Guo, Bo Yu, Jian Li, and Guang-Can Guo, "The orbital angular momentum of down-converted photons," Journal of Optics B: Quantum and Semiclassical Optics 6, 243-247 (200

[15] Jonathan Leach, Barry Jack, Jacqui Romero, Anand K. Jha, Alison M. Yao, Sonja Franke-Arnold, David G. Ireland, Robert W. Boyd, Stephen M. Barnett, and Miles J. Padgett, "Quantum correlations in optical Angle-Orbital angular momentum variables," Science 329, 662-665 (2010)

[16] Alois Mair, Alipasha Vaziri, Gregor Weihs, and Anton Zeilinger, "Entanglement of the orbital angular momentum states of photons," Nature 412, 313-316 (2001)

[17] H. Di Lorenzo Pires, H. C. B. Florijn, and M. P. van Exter, "Measurement of the spiral spectrum of entangled two-photon states," Physical Review Letters 104, 020505 (2010)

[18] M.J. Padgett and L. Allen, "The poynting vector in laguerre-gaussian laser modes," Optics Communications 121, 36-40 (1995). 